\title{
Evaluation of the American College of Radiology Thyroid Imaging, Reporting and Data System (Thyroid imaging reporting) scoring in thyroid Bethesda category on atypia and follicular lesion of uncertain significance patients
}

\author{
Oguz Catal ${ }^{1 *}$ (D), Bahri Ozer ${ }^{1}$ (D), Mustafa Sit ${ }^{1}$ (D), Hazal Yazgi ${ }^{1}$ (1)
}

\section{SUMMARY}

OBJECTIVE: Treatment and follow-up are controversial in patients whose thyroid fine needle aspiration biopsy (FNAB) is reported as atypia of undetermined significance and follicular lesion of uncertain significance (AUS/FLUS). We aimed the efficacy of the American College of Radiology Thyroid Imaging, Reporting and Data System (ACR TI-RADS) in preventing unnecessary thyroidectomies in patients with FNA cytology results as AUS/FLUS.

METHODS: In Bolu Abant Izzet Baysal University General Surgery Clinic, case series between 2017 and 2020 were analyzed with thyroid operated. Grouping was made according to the result of postoperative pathology: those with benign results after postoperative pathology were classified as Group 1, and those with malignant results after postoperative pathology were classified as Group 2.

RESULTS: As a result, 66 patients were found to be AUS/FLUS. A total of $28.8 \%$ of AUS/FLUS patients have been determined with cancer. In the statistical analysis of the ACR TI-RADS score between the groups, the ACR TI-RADS score in Group 1 patients (3.36) (SD 0.87) was significantly lower than that in Group 2 patients (4.11) (SD 1.04) ( $p=0.003)$. The distribution of the ACR TI-RADS scores of the patients in Group 2 was TR2: 2 (15.4\%) patients, TR3: 3 (25\%) patients, TR4: 5 (16.1\%), TR5: 9 (90\%) patients, respectively.

CONCLUSION: The ACR TI-RADS score was statistically significant in predicting malignancy in AUS/FLUS patients whose follow-ups and treatments are controversial, and the ACR TI-RADS has a limited role in preventing unnecessary thyroidectomies in patients with AUS/FLUS. KEYWORDS: Thyroid nodule. Thyroid neoplasms. Ultrasonography. Biopsy, fine-needle. Data systems.

\section{INTRODUCTION}

The incidence of thyroid cancer is increasing worldwide, and it is the most common cancer observed in the endocrine system ${ }^{1}$. An excellent prognosis is achieved with early diagnosis and surgery in thyroid malignancies ${ }^{2}$. Fine needle aspiration (FNA) cytology is a safe method that prevents unnecessary surgical procedures for a benign nodule 3,4 . In 2007, The Bethesda System for
Reporting Thyroid Cytopathology (TBSRTC) was proposed 5 . In TBSRTC, the third category has been defined as atypia of undetermined significance and follicular lesion of uncertain significance (AUS/FLUS) (BETHESDA 3). This category is a group of highly heterogeneous thyroid lesions with a high limit of cellularity. The "AUS/FLUS" category in TBSRTC caused controversy. The cases in this heterogeneous group include FNA

\footnotetext{
${ }^{1}$ Abant Izzet Baysal University Hospital, Department of General Surgery - Bolu, Turkey.

*Corresponding author: otuzogur@gmail.com

Conflicts of interest: the authors declare there are no conflicts of interest. Funding: none.

Received on December 29, 2020. Accepted on January 31, 2021.
} 
cytology results that are not as benign as can be clearly reported and are not as atypical as those in the Bethesda 4 and Bethesda 5 categories $^{6}$. In the literature, it has been stated in the thyroid FNA cytology reports that the rate of the use of AUS/FLUS is $2.1-18 \% 7$. In this group, the rate of malignancy in the pathological results of patients operated on varies between 6 and $48 \%{ }^{8}$. Uncertainty in predicting the risk of malignancy in AUS/FLUS leads to confusion in patient management?

It is desired that ultrasonography (USG) is a noninvasive method that shows the possibility of malignancy in thyroid nodules in the confidence interval of FNA cytology. In a recent study, the most specific features for thyroid carcinoma were stated to be microcalcifications, irregular borders, and changes in anteroposterior (AP)/ transverse diameter ${ }^{10}$. Using these features, the Thyroid Imaging, Reporting and Data System (TI-RADS) terminology was first used by Hovarth and his colleagues to determine the risk of malignancy by $\mathrm{USG}^{11}$. In addition, the recently implemented guidelines of the American College of Radiology (ACR) regulate the clinical management of patients with thyroid nodes with USG findings, and the guidelines help clinicians decide whether FNA cytology is required. In this approach, nodules with an ACR TI-RADS score lower than 3 are classified as benign (TR1)/nonsuspect (TR2), and biopsy is not recommended. In addition, if mild suspect nodules with an ACR TI-RADS score of 3 (TR3) are less than $25 \mathrm{~mm}$, the biopsy is not recommended, but the follow-up is recommended for nodules larger than $15 \mathrm{~mm}$ in this category ${ }^{12}$.

In this study, we investigated the efficacy of the ACR TI-RADS in preventing unnecessary thyroidectomies in patients with AUS/FLUS identified using FNA cytology results. Besides, we investigated the reliability of the ACR TI-RADS score in patients with AUS/FLUS.

\section{METHODS}

In Bolu Abant Izzet Baysal University (B.A.I.B.U) General Surgery Clinic, case series between 2017 and 2020 were analyzed with thyroid operated. Preoperative USG, thyroid FNA cytology results, and postoperative pathological results were examined. The preoperative thyroid FNA cytology results, the patients who were operated on, and the preoperative USG results containing data (i.e., composition, echogenicity, AP/ Transverse diameter, margin, and echogenic foci) suitable for ACR TI-RADS scoring were evaluated. Patients without preoperative FNA cytology results and ACR TI-RADS scoring in USG were excluded from this study. The ACR TI-RADS scores were obtained by calculating the ACR TI-RADS score of the nodule with the highest score in USG. The FNA biopsy (FNAB) was repeated in patients whose FNAB results were presented as AUS/FLUS, and when these results were the same, the patients were scheduled for thyroidectomy, The patients whose control FNAB results were reported as AUS/FLUS and who performed thyroidectomy were included in this study. The postoperative pathological results were obtained. Thyroid USGs were performed by two radiologists who were blinded to the study. Grouping was made according to the result of postoperative pathology: those with benign results after postoperative pathology were classified as Group 1 and those with the malignant result after postoperative pathology were classified as Group 2. Patients were grouped based on histology. The intent is to predict malignancy with TI-RADS.

\section{Statistics}

Whether variables were normally distributed or not was checked by the Kolmogorov-Smirnov test. While applying a $t$-test for variables with normal distribution, the Mann-Whitney $U$ test was used for the analysis of variables that do not show normal distribution. A crosstabs test was used to correlate between the ACR TI-RADS groups and its subgroups. The $\chi^{2}$ test was used for gender analysis. All the analyses were performed with the Statistical Package for Social Sciences 25.0 for Windows (SPSS Inc., Chicago, IL, USA), and the results with a level of $p<0.05$ were considered to be statistically significant.

\section{RESULTS}

Preoperative USG was performed in accordance with the ACR TI-RADS scoring. A total of 66 patients with preoperative FNA cytology and AUS/FLUS were included in this study. When the patients were grouped according to the result of postoperative pathology, there were 47 patients in Group 1 and 19 patients in Group 2. The classification of the patients in the groups according to the postoperative pathological results is shown in Table 1. Of the 259 patients with preoperative FNA cytology, the results of 66 patients were found to be AUS/FLUS. This constitutes $25.48 \%$ of all biopsy results. This rate is higher than the rates in the literature $(2.2-18 \%)$.

In the statistical analysis of the age values of 66 patients with AUS/FLUS according to the FNA cytology, the age of the patients in Group 1 was 47.51 (SD 9.54) and the age of the patients in Group 2 was 50.26 (SD 9.74) ( $\mathrm{p}=0.96)$. When the gender distributions are examined, there was no statistically significant difference $(\mathrm{p}=0.26)$.

In the statistical analysis of ACR TI-RADS score between the groups, the ACR TI-RADS score (3.36) (SD 0.87) in Group 1 patients was significantly lower than the ACR TI-RADS score (4.11) (SD 1.04) in Group 2 patients ( $\mathrm{p}=0.003$ ) (Table 2). It was observed that there were statistical differences between Group 1 and Group 2 in composition ( $\mathrm{p}=0.007$ ), echogenicity (0.009), and 
margin ( $\mathrm{p}=0.009$ ) subgroups that form the basis of ACR TI-RADS scoring. However, there were no statistical differences between Group 1 and Group 2 in shape $(\mathrm{p}=0.165)$ and echogenic foci $(\mathrm{p}=0.935)$ subgroups that form the basis of ACRTI-RADS scoring.

The distribution of patients according to TI-RADS groups is as follows: TR2 had 13/66 (19.7\%) patients, TR3 had 12/66 (18.2\%) patients, TR4 had 31/66 (47\%) patients, and TR5 had 10/66 (15.2\%) patients. It was observed that the most patients were in the TR4 group and the least patients were in the TR5 group. Comparing the groups and ACR TI-RADS scores of 66 patients with Bethesda 3 scores, ACR TI-RADS distributions, and numbers of patients, respectively, TR2 had $11(84.6 \%)$ patients in Group 1 and $2(15.4 \%)$ patients in Group 2; TR3 had 9 (75\%) patients in Group 1 and 3 (25\%) patients in Group 2; TR4 had 26 (83.9\%) patients in Group 1

Table 1. Distribution of patients by pathological results.

\begin{tabular}{l|l|c} 
Groups & Postoperative pathological results & $\mathrm{n}$ \\
\hline \multirow{5}{*}{ Group 1} & Nodular adenomatous hyperplasia & 17 \\
\cline { 2 - 3 } & Follicular hyperplasia & 8 \\
\cline { 2 - 3 } & Nodular colloidal goiter & 6 \\
\cline { 2 - 3 } & Adenomatous nodule & 4 \\
\cline { 2 - 3 } & Lymphocytic thyroid & 4 \\
\cline { 2 - 3 } & Follicular adenoma & 3 \\
\cline { 2 - 3 } & Subacute lymphocytic thyroid & 1 \\
\cline { 2 - 3 } & Hurthle cell adenoma & 2 \\
\cline { 2 - 3 } & Hashimoto thyroiditis & 1 \\
\cline { 2 - 3 } & Cavernous hemangioma & 10 \\
\hline \multirow{5}{*}{ Group 2} & Papillary thyroid carcinoma & 7 \\
\cline { 2 - 3 } & Minimal invasive follicular carcinoma & 1 \\
\cline { 2 - 3 } & Papillary microcarcinoma & 1 \\
\cline { 2 - 3 } & $\begin{array}{l}\text { Noninvasive follicular thyroid neoplasm } \\
\text { with papillary-like nuclear features } \\
\text { (NIFTP) }\end{array}$ & \\
\hline
\end{tabular}

and $5(16.1 \%)$ patients in Group 2; TR5 had $1(10 \%)$ patient in Group 1 and 9 (90\%) patients in Group 2 (Table 3). Cancer rates are very close between TR2 (15.4) and TR4 (16.1) due to the high number of benign patients in the TR 4 group.

When the ACR TI-RADS scores of 19 patients with malignant AUS/FLUS were evaluated according to the results of the postoperative pathology, no biopsy should have been performed in two patients in the TR2 category. According to the ACR TI-RADS algorithm, biopsies should not have been performed for three patients in the TR3 because the nodule sizes were below $2.5 \mathrm{~cm}$. In the TR 4 category, two patients whose nodule size was less than $1.5 \mathrm{~cm}$ should not have been biopsied and USG follow-up should have been performed. Thus, seven patients could not be diagnosed with thyroid cancer. In the TR5 category, FNAB was required according to the ACR TI-RADS system because the nodule sizes of all patients were above $1 \mathrm{~cm}$.

\section{DISCUSSION}

The follow-up and treatment of the results in the AUS/FLUS category are still controversial. While the average usage rate of AUS/FLUS ranges between 2.1 and $18 \%$ of all thyroid FNABs, the postoperative malignancy rate was reported to vary between 6 and $18 \%$ in AUS/FLUS ${ }^{7,8}$.

It is aimed to exclude cases that do not clearly exhibit benign features with the AUS/FLUS category. AUS/FLUS is a heterogeneous category with various abnormal features, but it is assumed to be at low risk of malignancy. It also includes samples with cellularity on the limits/low quality. Nodules with AUS/FLUS are often resected if suspicious clinical and USG features are present and there are abnormal results in recurrent FNA cytology. Resection is not performed in patients who are reported as benign in recurrent FNA cytology ${ }^{13}$. The USG of thyroid nodules is the most effective diagnostic tool to predict malignancy and select lesions that require further evaluation ${ }^{14}$. The USG-FNA cytology is the most cost-effective procedure that provides useful diagnostic information about advanced

Table 2. Test results of American College of Radiology Thyroid Imaging, Reporting and Data System and subgroups.

\begin{tabular}{l|c|c|c} 
& Group 1 $(\mathrm{n}=47)$ & Group 2 $(\mathrm{n}=19)$ & p-value \\
\hline TI-RADS & 3.36 SD 0.87 & 4.11 SD 1.04 & 0.003 \\
\hline Composition & $1(0-2)$ & $1(1-2)$ & 0.007 \\
\hline Echogenicity & $0(0-3)$ & $3(0-3)$ & 0.009 \\
\hline Shape & $0(0-2)$ & $0(0-2)$ & 0.165 \\
\hline Margin & $0.060(0.03-0.15)$ & $0.057(0.03-0.10)$ & 0.009 \\
\hline Echogenic foci & $0(0-3)$ & $0(0-3)$ & 0.935 \\
\hline
\end{tabular}

TI-RADS: Thyroid Imaging Reporting and Data System. 
Table 3. Comparison of groups with American College of Radiology Thyroid Imaging, Reporting and Data system.

\begin{tabular}{|c|c|c|c|c|c|c|c|}
\hline \multirow{3}{*}{ TI-RADS } & \multirow{3}{*}{$\begin{array}{c}\% \text { of total } \\
\text { patients }\end{array}$} & \multicolumn{6}{|c|}{ Patients } \\
\hline & & \multicolumn{2}{|c|}{ Group 1} & \multicolumn{2}{|c|}{ Group 2} & \multicolumn{2}{|c|}{ Total } \\
\hline & & $n$ & $(\%)$ & $n$ & $(\%)$ & $n$ & $(\%)$ \\
\hline $\operatorname{Tr} 2$ & $13 / 66(19.7)$ & 11 & 84.6 & 2 & 15.4 & 13 & 100 \\
\hline $\operatorname{Tr} 3$ & $12 / 66(18.2)$ & 9 & 75.0 & 3 & 25.0 & 12 & 100 \\
\hline Tr4 & $31 / 66(47)$ & 26 & 83.9 & 5 & 16.1 & 31 & 100 \\
\hline $\operatorname{Tr} 5$ & $10 / 66(15.2)$ & 1 & 10 & 9 & 90 & 10 & 100 \\
\hline
\end{tabular}

TI-RADS: Thyroid Imaging, Reporting and Data System.

clinical management ${ }^{15}$. The clinical and practical purpose of this diagnostic tool is to reduce the number of unnecessary surgical procedures in patients with benign nodules and to identify people at high risk of malignancy. While 47 of the 66 patients with AUS/FLUS in our study were benign as a result of postoperative pathology, $19(28.78 \%)$ of the patients were reported as malignant. The rate of $28.78 \%$ that we reported was found to be higher than the rate of $6-18 \%{ }^{7}$ AUS/FLUS malignancy, which is stated in the world literature.

There are many guidelines regarding the USG evaluation of thyroid nodules ${ }^{16}$. Several USG-based methods were developed to classify the risk of malignancy of thyroid nodules in adults. Two of these methods are Kwak TI-RADS and ACR TI-RADS (which is officially stated by the ACR in 2017 for the management of thyroid nodules) ${ }^{12}$. ACR TI-RADS is based on evaluating the USG properties of nodules in five categories (i.e., composition, echogenicity, shape, shape, and echogenic foci) where each category is given $0-3$ points. While each of the features in the first four categories has a single score derived from exclusive choices, there may be more than one feature in the echogenic foci category. In ACR TI-RADS, the scores are given for all USG features in a nodule, i.e., the more suspicious features get higher scores. In other words, the possibility of malignancy associated with each feature is weighted and considered differently. Along with the maximum diameter of the nodule, the TR level recommends that FNA cytology, follow-up by USG, or any other procedure should not be performed to the patient.

Total scores determine the level of ACR TI-RADS of the nodule, ranging from TR1 (benign) to TR5 (suspicious of high malignancy $)^{12}$. Compliance with ACR TI-RADS will result in less benign nodule biopsy, as the threshold diameters for mild and moderately suspect nodules (TR3 and TR4) are larger than other systems. However, it will result in less malignant nodule cytology. Therefore, ACR TI-RADS recommends observation and following of some nodules that do not meet the size criteria for FNA cytology ${ }^{17}$.

In our study, we showed that ACR TI-RADS was statistically significant in predicting malignancy in patients with AUS/FLUS. We concluded that unnecessary surgery can be prevented by the ACR TI-RADS assessment in patients with AUS/FLUS. Although it was statistically significant, when examined proportionally, it was observed that only TI-RADS appeared as a robust tool in predicting malignancy with only the TR5 group (90\%), but this was not valid for other TR groups. Consequently, it was concluded that seven patients with AUS/FLUS, whose postoperative results were reported as malignant, could not be diagnosed. This number constitutes a high rate of $36.8 \%$ of 19 patients with AUS/FLUS who were malignant according to the results of pathology.

All of the patients were operated on by evaluating preoperative FNAB. This study has two limitations. One is that this is a retrospective study, and the other is that the number of cases is limited in our study.

\section{CONCLUSION}

The ACR TI-RADS score was statistically significant in predicting malignancy in AUS/FLUS patients whose follow-ups and treatments are controversial, and ACR TI-RADS has a limited role in preventing unnecessary thyroidectomies in patients with AUS/FLUS.

\section{AUTHORS" CONTRIBUTIONS}

OC: Conceptualization, Data curation, Formal Analysis, Writing - original draft. MS: Writing - original draft, Writing - review \& editing. BO: Data curation, Formal Analysis. HY: Data curation. 


\section{REFERENCES}

1. Cho BY, Choi HS, Park YJ, Lim JA, Ahn HY, Lee EK, et al. Changes in the clinicopathological characteristics and outcomes of thyroid cancer in Korea over the past four decades. Thyroid. 2013;23(7):797-804. https://doi.org/10.1089/thy.2012.0329

2. Goldstein RE, Netterville JL, Burkey B, Johnson JE. Implications of follicular neoplasms, atypia, and lesions suspicious for malignancy diagnosed by fine-needle aspiration of thyroid nodules. Ann Surg. 2002;235(5):656-62. https://doi. org/10.1097/00000658-200205000-00007

3. VanderLaan PA, Marqusee E, Krane JF. Clinical outcome for atypia of undetermined significance in thyroid fine-needle aspirations: should repeated FNA be the preferred initial approach? Am J Clin Pathol. 2011;135(5):770-5. https://doi. org/10.1309/AJCP4P2GCCDNHFMY

4. American Thyroid Association (ATA) Guidelines Taskforce on Thyroid Nodules and Differentiated Thyroid Cancer, Cooper DS, Doherty GM, Haugen BR, Kloos RT, Lee SL, et al. Revised American Thyroid Association management guidelines for patients with thyroid nodules and differentiated thyroid cancer: the American Thyroid Association (ATA) guidelines taskforce on thyroid nodules and differentiated thyroid cancer. Thyroid. 2009;19(11):1167-214. https://doi.org/10.1089/thy.2009.0110

5. Baloch ZW, LiVolsi VA, Asa SL, Rosai J, Merino MJ, Randolph $G$, et al. Diagnostic terminology and morphologic criteria for cytologic diagnosis of thyroid lesions: a synopsis of the national cancer institute thyroid fine-needle aspiration state of the science conference. Diagn Cytopathol. 2008;36(6):425-37. https://doi.org/10.1002/dc.20830

6. Horne MJ, Chhieng DC, Theoharis C, Schofield K, Kowalski $\mathrm{D}$, Prasad $\mathrm{ML}$, et al. Thyroid follicular lesion of undetermined significance: Evaluation of the risk of malignancy using the two-tier sub-classification. Diagn Cytopathol. 2012;40(5):410-5. https://doi.org/10.1002/dc.21790

7. Baloch ZW, Cibas ES, Clark DP, Layfield $\sqcup$, Ljung BM, Pitman MB, et al. The National Cancer Institute Thyroid fine needle aspiration state of the science conference: a summation. Cytojournal. 2008;5(16):6. https://doi.org/10.1186/1742-6413-5-6

8. Bongiovanni M, Krane JF, Cibas ES, Faquin WC. The atypical thyroid fine-needle aspiration: past, present, and future. Cancer Cytopathol. 2012;120(2):73-86. https://doi.org/10.1002/ cncy.20178
9. Nagarkatti SS, Faquin WC, Lubitz CC, Garcia DM, Barbesino G, Ross DS, et al. Management of thyroid nodules with atypical cytology on fine-needle aspiration biopsy. Ann Surg Oncol. 2013;20(1):60-5. https://doi.org/10.1245/s10434-012-2601-2

10. Haugen BR, Alexander EK, Bible KC, Doherty GM, Mandel SJ, Nikiforov YE, et al. 2015 American Thyroid Association management guidelines for adult patients with thyroid nodules and differentiated thyroid cancer: the American Thyroid Association guidelines task force on thyroid nodules and differentiated thyroid cancer. Thyroid. 2016;26(1):1-133. https://doi.org/10.1089/thy.2015.0020

11. Horvath E, Majlis S, Rossi R, Franco C, Niedmann JP, Castro A, et al. An ultrasonogram reporting system for thyroid nodules stratifying cancer risk for clinical management. J Clin Endocrinol Metab. 2009;94(5):1748-51. https://doi.org/10.1210/jc.20081724

12. Tessler FN, Middleton WD, Grant EG, Hoang JK, Berland LL, Teefey SA, et al. ACR thyroid imaging, reporting and data system (TI-RADS): white paper of the ACR TI-RADS committee. J Am Coll Radiol. 2017;14(5):587-95. https://doi.org/10.1016/j. jacr.2017.01.046

13. Cibas ES and Ali SZ. The 2017 Bethesda system for reporting thyroid cytopathology. Thyroid. 2017;27(11):1341-6. https:// doi.org/10.1089/thy.2017.0500

14. Chng CL, Kurzawinski TR, Beale T. Value of sonographic features in predicting malignancy in thyroid nodules diagnosed as follicular neoplasm on cytology. Clin Endocrinol (Oxf). 2015;83(5):711-6. https://doi.org/10.1111/cen.12692

15. Kaliszewski K, Zubkiewicz-Kucharska A, Wojtczak B, StrutyńskaKarpińska M, Zaleska-Dorobisz U, Leśków E. Ultrasound guided fine-needle aspiration biopsy of thyroid nodules: Does radiologist assistance decrease the rate of unsatisfactory biopsies? Adv Clin Exp Med. 2016;25(1):93-100. https://doi. org/10.17219/acem/60084

16. Ha EJ, Baek JH, Na DG. Risk stratification of thyroid nodules on ultrasonography: current status and perspectives. Thyroid. 2017;27(12):1463-8. https://doi.org/10.1089/thy.2016.0654

17. Tessler FN, Middleton WD, Grant EG. Thyroid imaging reporting and data system (TI-RADS): a user's guide. Radiology. 2018;287(1):29-36. https://doi.org/10.1148/radiol.2017171240 